\title{
Caracterização biométrica de tubarões do gênero Sphyrna (Griffith \& Smith, 1834) no litoral de Sergipe
}

\author{
Jéssica Barros Andrade * \\ Daniela Nascimento Santos \\ Maraisa de Oliveira Silva \\ Thays Brito Reis Santos \\ Thiago Silveira Meneses \\ Andressa Sales Coelho \\ Universidade Tiradentes, Campus Farolândia, Aracaju, CEP 49032-490, Aracaju - SE, Brasil \\ * Autor para correspondência \\ jessicabandrade@hotmail.com
}

Submetido em 01/10/2015

Aceito para publicação em 29/03/2016

\section{Resumo}

Os tubarões, quimeras e raias pertencentes ao grupo de Elasmobrânquios da Classe dos Condrichthyes, possuem uma maturação e desenvolvimento lento e são animais bastante capturados em pescas acompanhantes, tornando-se animais vulneráveis à extinção. Seis espécies do gênero Sphyrna são encontradas no Brasil, em zona costeira-oceânica, três destas foram estudadas: Sphyrna lewini, S. mokarran e S. tiburo. O estudo teve como objetivo obter a biometria, identificação do sexo e o estágio de desenvolvimento sexual do gênero Sphyrna na pesca artesanal de Aracaju - SE. Para o trabalho foram utilizados animais da coleção do GEES e animais frescos adquiridos da pesca local. Sphyrna tiburo foi encontrada apenas na coleção. As espécies S. mokarran e S. lewini obtiveram mais indivíduos neonatos e machos, e $S$. tiburo, fêmeas e juvenis. Para as espécies frescas, a medida peitoral-pélvica foi maior em fêmeas e pélvico-anal maior nos machos de S. lewini. O peso foi maior na S. lewini, e para o comprimento total, largura da cabeça e comprimento interdorsal não houve diferença entre os espécimes. Os parâmetros medidos não apresentaram resultados significativos, o que pode estar relacionado à característica da pesca, que apresentou muitos indivíduos imaturos.

Palavras-chave: Biometria; Condrichthyes; Elasmobrânquios; Sphyrna

\section{Abstract}

Biometric characterization of sharks of the genus Sphyrna (Griffith \& Smith, 1834) on the coast of Sergipe. Sharks, rays and chimeras belonging to the subclass Elasmobranchii of the class Chondrichthyes have a slow maturation and development, and they are animals often caught as by-catch, making them vulnerable to extinction. Six species of the genus Sphyrna are found in Brazil, in the coastal-oceanic zone, three of which were studied: Sphyrna lewini, S. mokarran and S. tiburo. This study aimed to evaluate the biometrics, sex and stage of sexual development of the genus Sphyrna in artisanal fishing in Aracaju (SE). The animals used included specimens from the GEES collection and fresh ones acquired from local fisheries. S. tiburo was found only 
in the collection. The species $S$. mokarran and S. lewini included more newborn individuals and males and $S$. tiburo more females and juveniles. In fresh species, pectoral-pelvic length was greater in females, and pelvicanal length was greater in males of $S$. lewini. Weight was greatest in S. lewini, while there was no difference in total length, head width and interdorsal length between specimens. The parameters measured did not show significant results that could be related to by-catch with many immature individuals.

Key words: Biometrics; Chondrichthyes; Elasmobranchii; Sphyrna

\section{Introdução}

A família Sphyrnidae (Gill, 1872) tem como representante as diferentes espécies de tubarão-martelo presentes em dois gêneros: Eusphyra, com uma espécie somente (restrita ao Indo-Pacífico) e Sphyrna, com sete espécies, das quais seis ocorrem no litoral brasileiro: S. zygaena, S. tiburo, S. tudes, S. media, S. mokarran e $S$. lewini (GADIG, 1994). A diferença na morfologia entre as espécies é frequentemente embasada, sobretudo, na morfologia da cabeça, observando-se seu contorno anterior, presença de sulco nasal interno e padrão de distribuição dos poros associados às ampolas de Lorenzini (SADOWSKY, 1965; GILBERT, 1967).

Os indivíduos dessas espécies são mais abundantes nos meses de primavera e verão (SBEEL, 2005). O gênero apresenta uma ampla distribuição global e com isso ocupa hábitats dos mais variados, desde as zonas costeiras às oceânicas e de regiões tropicais às temperadas (BEZERRA, 2013). Dependendo do estágio de desenvolvimento, os espécimes vão se aprofundando no ambiente, ou seja, neonatos são mais encontrados em águas costeiras, enquanto os adultos, em águas mais profundas (KOTAS, 2004).

Espécies costeiras e de pequeno porte como $S$. tudes, S. media e S. tiburo já foram encontradas em toda a costa brasileira, porém hoje são mais frequentes nas regiões Norte e Nordeste. As espécies de grande porte como S. lewini, S. zygaena e S. mokarran ocorrem em toda a costa, ressaltando-se que este último ocorre mais nas regiões Norte e Nordeste (SADOWSKY, 1965; FIGUEIREDO, 1977; GADIG, 2001; SOTO, 2001).

Geralmente são de médio e grande porte, variando de 1,3 a 6 m de comprimento. São vivíparos placentários, com nascimento de até 43 filhotes por gestação, e alimentam-se principalmente de teleósteos, mas também apresentam na sua dieta outros elasmobrânquios, crustáceos, moluscos e invertebrados (GADIG, 1994).

Quattro et al. (2013) descreveram, na costa da Carolina do Sul, mais uma espécie deste gênero, $S$. gilberti, muito semelhante ao $S$. lewini, em que a largura da cabeça representa entre 25 a $32 \%$ do comprimento total do corpo, sendo em geral indivíduos neonatos menores que os $S$. lewini, podendo-se classificar estes como adultos de menor tamanho também.

Sphyrna lewini é considerada a espécie mais abundante do gênero e habita águas mais profundas, acima de 80 m (COMPAGNO, 1984). Normalmente, pode ser confundida com as outras espécies do gênero, não sendo possível saber a classificação exata do seu grau de extinção. No ano de 2004, foi considerada a espécie mais criticamente ameaçada entre os tubarões, devido ao comércio das barbatanas não tratadas, que têm alto valor econômico comparado com as outras espécies capturadas, e isso acaba por diminuir ainda mais os indivíduos (KOTAS, 2004).

A biodiversidade dos elasmobrânquios vem sendo bastante afetada pela pesca artesanal e industrial devido à falta de informação dos pescadores sobre a importância das espécies e os impactos causados pela sua captura (BONFIL, 1994; LESSA et al., 1999).

Devido à pesca excessiva, várias espécies de tubarões estão ameaçadas de extinção e compõem a Lista Vermelha da União Internacional para a Conservação da Natureza (IUCN). As espécies $S$. lewini e $S$. tiburo encontram-se classificadas como criticamente em perigo (CR), enquanto $S$. mokarran encontra-se classificada como em perigo (EN) na Lista Nacional Oficial de Espécies da Fauna Ameaçadas de Extinção (BRASIL, 2014). Segundo a classificação da IUCN (2015), as duas primeiras encontram-se em perigo (EN), e a terceira, em situação de pouca preocupação (LC). 
A captura de elasmobrânquios no Brasil foi considerada por muito tempo uma pesca incidental mesmo tendo registros da renda comercial de sua carne e subprodutos. Hoje já se pode observar a ocorrência de embarcações destinadas somente à pesca de tubarões, devido à valorização dos subprodutos tanto no mercado comercial interno quanto no exterior (SBEEL, 2005).

Normalmente, observam-se três tipos de pesca: a pesca artesanal, em que se obtêm mais pescados de pequeno porte, neonatos e juvenis, e que apresenta suma importância para o país, pois compõe o alimento das populações de baixa renda; a pesca industrial, em que ocorre a captura de indivíduos de grande porte para a retirada das nadadeiras que são consumidas tanto nacional como internacionalmente, e, neste caso, as embarcações são destinadas para espécies-alvo com valor econômico e por último, a pesca esportiva por mergulho, que pode levar a redução das populações de elasmobrânquios (MENESES et al., 2011).

Essas modalidades de pesca necessitam de um maior controle e monitoramento associados a ações de proteção e conservação da biodiversidade. Outro fator que ameaça a biodiversidade dos tubarões é o impacto das regiões costeiras e estuarinas devido à degradação desses ambientes, que muitas vezes são utilizados como local de berçário e parto para diversas espécies (LESSA et al., 1999).

Diante desse cenário, no ano de 2000, foi elaborado o "Plano de Ações para a conservação e o Manejo dos estoques de Elasmobrânquios no Brasil" cujos objetivos foram ampliar o conhecimento e a proteção das espécies, principalmente aquelas vulneráveis e ameaçadas e seus hábitats; assegurar a exploração sustentável em curto e longo prazo e que capturas incidentais ou dirigidas sejam sustentáveis. As atividades pesqueiras, sua fiscalização e medidas necessárias para o manejo dessas atividades ficaram sob responsabilidade do Instituto Brasileiro de Meio Ambiente (IBAMA) e do Ministério da Agricultura e do Abastecimento (MAA) (SBEEL, 2005).

Os tubarões-martelo são migratórios e sazonais e vêm sendo capturados como fauna acompanhante de diversas formas de pesca na região Sul e Sudeste, e têm sido alvo nas pescas de emalhe e espinhel de superfície. Normalmente são coletados das redes sem vida provavelmente devido à grande quantidade de órgãos sensoriais existentes na cabeça, gerando um alto nível de estresse para os animais (BONFIL, 1994; SBEEL, 2005).

O Plano de Ação é valido para todas as regiões do Brasil, contudo o Nordeste é a região que mais tem deficiência de estudos sobre os tipos de pesca e ocorrência de espécies. Segundo informações discutidas no SBEEL (2005), nessa região não é observada a pesca direcionada para elasmobrânquios, sendo os espécimes capturados de caráter incidental na pesca de teleósteos o que comprova que muitas espécies usam a costa da região Nordeste como berçário.

O objetivo do presente trabalho foi caracterizar biometricamente espécimes fixados e frescos do gênero Sphyrna, oriundos da pesca artesanal de Aracaju/SE, quanto ao seu peso, sexo, estágio de desenvolvimento sexual e as características mais representativas, como: largura da cabeça, comprimento total, comprimento interdorsal, comprimento peitoral-pélvico e comprimento pélvico-anal.

\section{Material e Métodos}

\section{Área de estudo}

O trabalho foi realizado no litoral da cidade de Aracaju, Sergipe. O estado apresenta um litoral com $163 \mathrm{~km}$ de extensão caracterizado por um relevo de depressões, várzeas e dunas. Além de uma vegetação típica de coqueirais, mangues e restingas, característica da região de praias, no estado também se encontra os biomas Mata Atlântica e Caatinga (SANTOS; VILAR, 2012).

Aracaju possui diversos terminais pesqueiros, muitos de pequena escala, e para a presente pesquisa os exemplares de tubarões foram adquiridos nas praias do Mosqueiro e Atalaia, região sul da cidade (Figura 1). 
FIGURA 1: Mapa da região praieira de Aracaju.

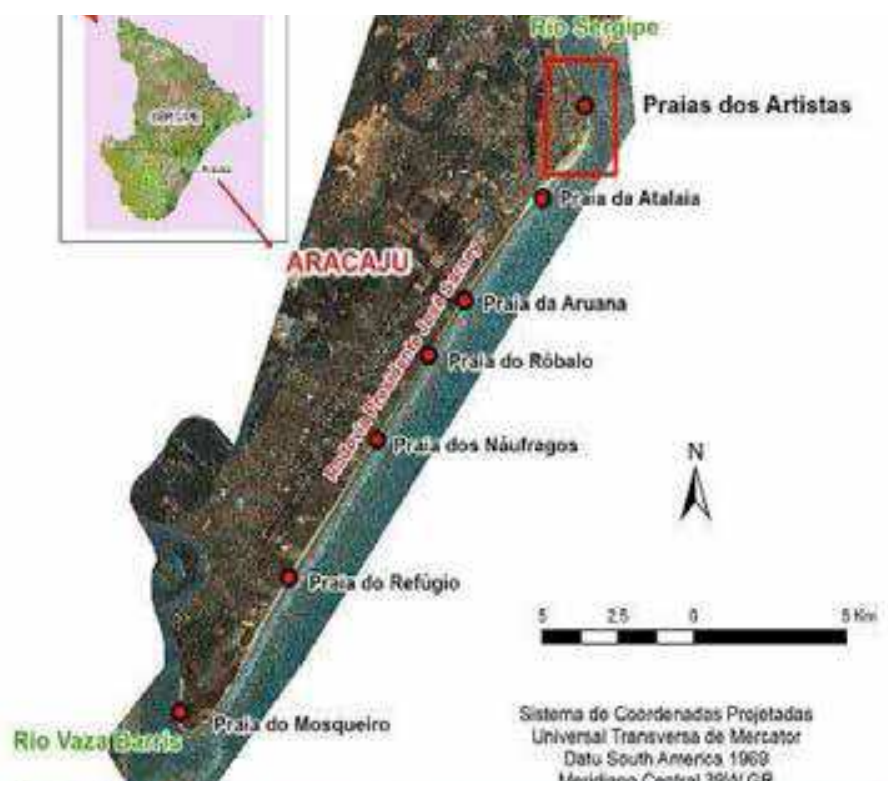

Fonte: https://ri.ufs.br/bitstream/123456789/964/1/ParametrosGranulometricos.pdf

\section{Aquisição de exemplares}

A amostra da pesquisa foi formada por animais fixados oriundos da coleção zoológica do GEES e animais frescos provenientes da pesca.

\section{Exemplares fixados - Coleção Zoológica/GEES}

O Grupo de Estudo de Elasmobrânquios de Sergipe (GEES), localizado na Universidade Tiradentes, possui uma coleção zoológica que teve início no ano de 2001, visando servir como depositária dos espécimes capturados no estado. A última atualização da coleção foi em agosto de 2013, em que foram registrados 530 exemplares catalogados em 213 lotes devidamente cadastrados no banco de dados do GEES, sendo $88 \%$ dessa amostra oriunda de Sergipe, e 12\%, dos estados da Bahia, Pará, Rio Grande do Sul, São Paulo e Tocantins por meio de permutas e doações. A coleção é composta por espécimes inteiros, cabeça, crânios, arcadas dentárias e embriões conservados em meio seco e úmido, formol e álcool 70\%. Após a aquisição e identificação dos exemplares, todos foram dissecados, fixados e então conservados em potes grandes de plástico com formol (GEES, 2013).
A coleção zoológica do GEES possui 14 exemplares da família Sphyrnidae, utilizados na presente pesquisa e pertencentes a três espécies: Sphyrna lewini, Sphyrna mokarran e Sphyrna tiburo (Tabela 1).

TABELA 1: Número de exemplares e anos de coleta de espécies do gênero Sphyrna presentes na coleção zoológica do GEES.

\begin{tabular}{lcc}
\multicolumn{1}{c}{ Espécie } & № de indivíduos & Anos de coleta \\
\hline Sphyrna tiburo & 5 & $2002,2006,2007$ \\
Sphyrna mokarran & 4 & $2001,2004,2006$ \\
Sphyrna lewini & 5 & $2001,2004,2005$ \\
\hline
\end{tabular}

Fonte: Banco de dados GEES.

Os animais da coleção analisados nesta pesquisa foram adquiridos principalmente na região do Mosqueiro, contudo alguns foram identificados como capturados em Sergipe, no Litoral Sul e na Praia da Caueira. Os artefatos mais utilizados para a pesca desses exemplares foram as redes de emalhar e de arrasto de fundo, sendo a primeira a mais utilizada. O banco de dados possui as seguintes informações para esses espécimes: profundidade de captura, tipo de rede utilizada na pescaria, estágio de desenvolvimento sexual, sexo e comprimento total do animal. 


\section{Exemplares frescos}

Novos exemplares foram adquiridos no período de janeiro a abril de 2015 e são provenientes de embarcações que desembarcam nas praias de Atalaia e Mosqueiro. A embarcação do Mosqueiro foi classificada como de pequeno porte, com $8 \mathrm{~m}$ de comprimento e $80 \mathrm{~cm}$ de largura, e utilizava rede de espera com $600 \mathrm{~m}$ de comprimento e malha de nylon de $40 \mathrm{~cm}$. A profundidade de captura variou de 6 a $8 \mathrm{~m}$ (comunicação pessoal, embarcação Gavião).

Os indivíduos obtidos no terminal pesqueiro da Atalaia foram capturados por embarcações de pequeno porte, com $7 \mathrm{~m}$ de comprimento e 1,40 de largura, que utilizam rede de espera de aproximadamente 1.200 $\mathrm{m}$, com malha de nylon de $40 \mathrm{~cm}$ e a profundidade de coleta é de 4 a 5 m (comunicação pessoal, embarcação Jupira e Diana).

\section{Coleta de dados biométricos \\ Exemplares fixados - Coleção Zoológica/GEES}

Para os exemplares provenientes da coleção foram adquiridas cinco medidas biométricas conforme
Compagno (2001) (Tabela 2). O peso não foi identificado devido ao tempo de fixação dos animais e porque alguns não tinham órgãos internos, impossibilitando tal análise. A biometria dos espécimes fixados foi realizada como treinamento na coleta de dados biométricos e serviu de informação extra, acrescentada ao banco de dados do GEES, porém não foi utilizada na análise estatística dos dados, devido ao fato de esses exemplares já estarem há muito tempo fixados e sem estruturas internas, podendo assim influenciar nos resultados.

\section{Exemplares frescos}

Para os exemplares adquiridos no período de janeiro a abril de 2015, foram tomadas as medidas biométricas segundo Compagno (2001) (Tabela 2), utilizando-se fita métrica para medição do comprimento total (Figura 3) e paquímetro (com precisão de $\mathrm{cm}$ ) para as demais medidas (Figuras 2, 4 e 5). Todas as medidas foram obtidas em centímetros. Adicionalmente, foram identificados: espécie, sexo, estágio de desenvolvimento segundo Compagno (1984) e Lessa e Menni (1998) (Tabela 3) e peso. Posteriormente, todos os indivíduos foram fixados em formol e depositados na coleção do GEES.

TABELA 2: Tipo, categoria, sigla e definição das medidas biométricas realizadas para tubarões do gênero Sphyrna adquiridos no litoral de Sergipe.

\begin{tabular}{|c|c|c|c|}
\hline Tipo de medida & $\begin{array}{l}\text { Categoria da } \\
\text { medida }\end{array}$ & Sigla & Definição \\
\hline Comprimento total & $\begin{array}{l}\text { Corpórea } \\
\text { Longitudinal }\end{array}$ & $\mathrm{CT}$ & $\begin{array}{l}\text { Medida do focinho até a ponta da nadadeira caudal } \\
\text { (Figura } 3 \text { ). }\end{array}$ \\
\hline $\begin{array}{l}\text { Comprimento do espaço } \\
\text { interdorsal }\end{array}$ & Espaços Corpóreos & EID & $\begin{array}{l}\text { Medida da inserção da primeira nadadeira dorsal até a } \\
\text { origem da segunda dorsal (Figura 5). }\end{array}$ \\
\hline Comprimento peitoral-pélvico & Espaços Corpóreos & EPP & $\begin{array}{l}\text { Medida da inserção da nadadeira peitoral até a origem } \\
\text { da nadadeira pélvica. }\end{array}$ \\
\hline Comprimento pélvico-anal & Espaços Corpóreos & EPA & $\begin{array}{l}\text { Medida da inserção da nadadeira pélvica até a origem } \\
\text { da anal. }\end{array}$ \\
\hline Largura da cabeça & Cabeça & HDW & $\begin{array}{l}\text { Medida referente à distância de um olho a outro } \\
\text { (Figura 4). }\end{array}$ \\
\hline
\end{tabular}

Fonte: Compagno (2001). 
FIGURA 2: Formato e largura da cabeça dos espécimes Sphyrna lewini, S. tiburo, S. mokarran pertencentes à coleção zoológica do GEES. Foto: Daniela Nascimento.

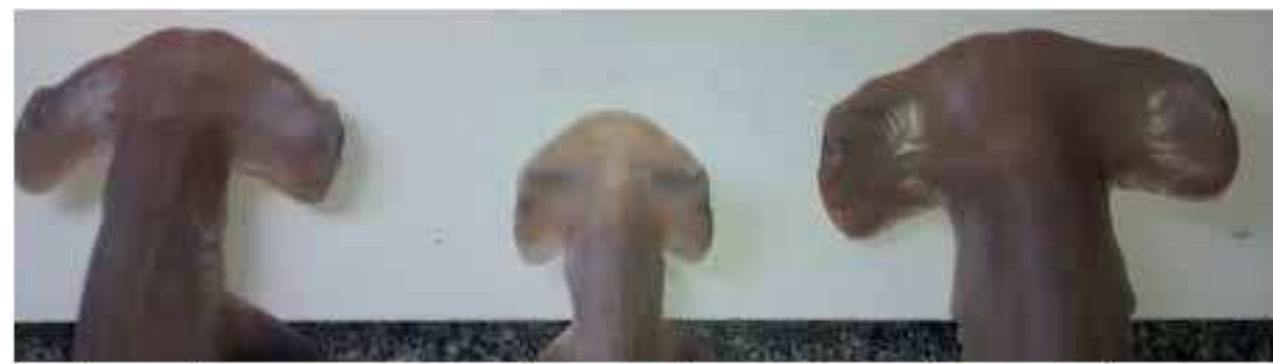

Sphyrna lewini

Sphyrna tiburo

Sphyrna mokarran

FIGURA 3: Foto referente à medida de Comprimento Total de um espécime de Sphyrna lewini. Foto: Daniela Nascimento.

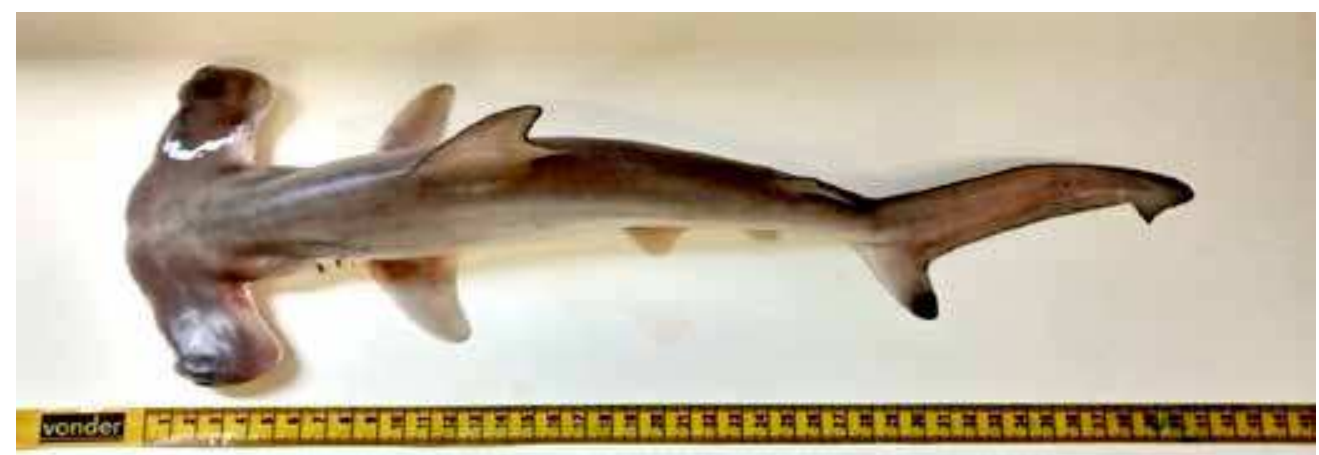

FIGURA 4: Foto referente à medida de largura da cabeça, em um exemplar de Sphyrna lewini. Foto: Jéssica Andrade.

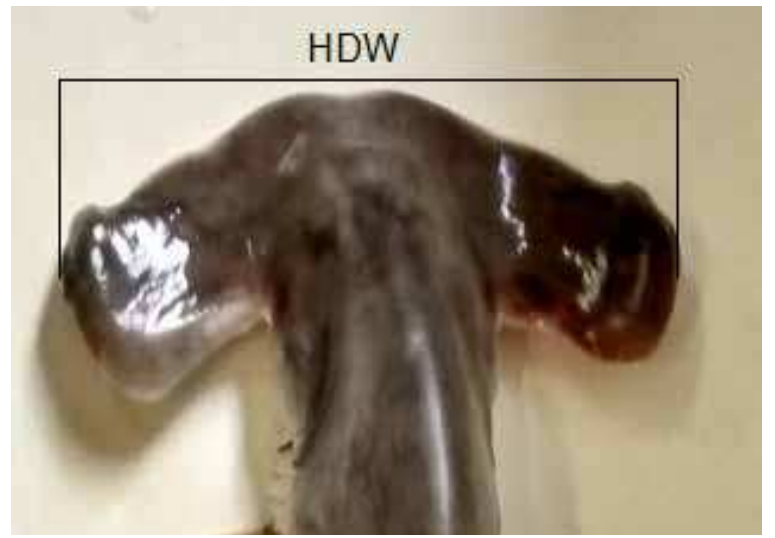

FIGURA 5: Foto referente à medida de comprimento interdorsal de um exemplar Sphyrna lewini. Foto: Daniela Nascimento.

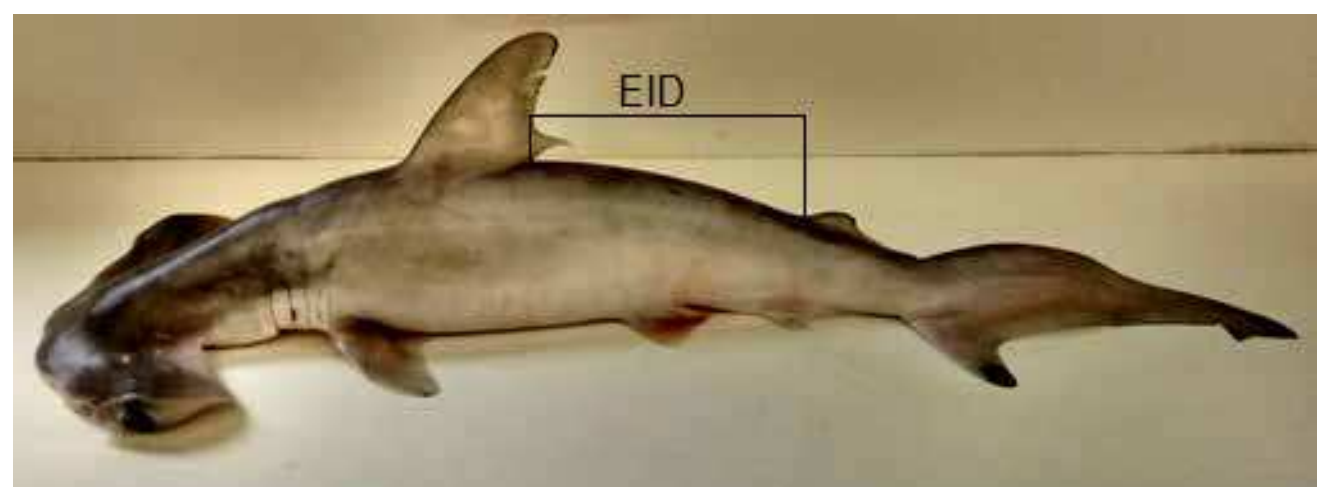


TABELA 3: Classificação e definição dos estágios de desenvolvimento sexual dos elasmobrânquios do gênero Sphyrna adquiridos no litoral de Sergipe.

\begin{tabular}{c|l}
$\begin{array}{c}\text { Estágio de } \\
\text { desenvolvimento } \\
\text { sexual }\end{array}$ & \multicolumn{1}{c}{ Definição } \\
\hline Neonato & $\begin{array}{l}\text { Cicatriz do cordão umbilical } \\
\text { totalmente aberta entre as nadadeiras } \\
\text { peitorais. }\end{array}$ \\
\hline Juvenil & $\begin{array}{l}\text { Cicatriz do cordão umbilical visível, } \\
\text { porém fechada, ou ausente. Nos } \\
\text { machos clásper flexível e nas fêmeas } \\
\text { ovários pouco evidentes. }\end{array}$ \\
\hline Adulto & $\begin{array}{l}\text { Não existe mais cicatriz e o clásper, } \\
\text { nos machos, é rígido e nas fêmeas } \\
\text { ovócitos no ovário e ovos ou embriões } \\
\text { no útero. }\end{array}$ \\
\hline
\end{tabular}

Fonte: Compagno (1984); Lessa e Menni (1998); Castro (1993) e Simpfendorfer e Milward (1993).

\section{Análise dos dados}

Os dados coletados foram armazenados no MSExcel e analisados de forma descritiva para todos os espécimes (fixados e frescos). Foram calculadas as frequências de: sexo por espécie, estágio de desenvolvimento por espécie e sexo e estágio de desenvolvimento por espécie. Adicionalmente, para os exemplares frescos, foi calculada a média de comprimento total e peso por sexo para cada espécie. A significância entre espécies e por sexo entre gêneros foi testada para as medidas: Largura da cabeça, Espaço interdorsal, Comprimento total, Comprimento peitoralpélvico e Comprimento pélvico-anal. Foi utilizado o teste de Wilcoxon para as duas primeiras medidas e o teste-T de Student para as demais com nível de significância de 5\%. O programa utilizado foi o BioEstat (versão 5.0).

\section{Resultados}

O GEES possui um projeto sobre a pesca artesanal de elasmobrânquios que vem sendo desenvolvido desde abril de 2014. A Tabela 4 mostra a representatividade do gênero Sphyrna dentro da amostra total do projeto referente à pesca artesanal de tubarões no período de abril de 2014 a abril de 2015.

A Tabela 5 mostra o tamanho da amostra incluindo indivíduos fixados e frescos, totalizando 39 espécimes. A espécie mais abundante foi o Sphyrna lewini,

TABELA 4: Número de indivíduos, sexo, estágio de desenvolvimento e frequência de ocorrência do gênero Sphyrna na pesca artesanal de tubarões em Sergipe de abril de 2014 a abril de 2015.

\begin{tabular}{|c|c|c|c|c|c|c|}
\hline \multirow{2}{*}{ Espécies } & \multirow{2}{*}{$\begin{array}{c}\text { № de } \\
\text { Indivíduos }\end{array}$} & \multirow{2}{*}{$\begin{array}{c}\text { Frequência } \\
\%\end{array}$} & \multicolumn{2}{|c|}{$\begin{array}{c}\text { Estágio de } \\
\text { desenvolvimento sexual }\end{array}$} & \multicolumn{2}{|c|}{ Sexo } \\
\hline & & & Neonato & Juvenil & Macho & Fêmea \\
\hline Carcharhinus porosus & 4 & $5,3 \%$ & 1 & 3 & 0 & 4 \\
\hline Rhizoprionodon lalandii & 29 & $8,2 \%$ & 12 & 17 & 21 & 8 \\
\hline Rhizoprionodon porosus & 10 & $13,2 \%$ & 8 & 2 & 2 & 8 \\
\hline Rhizoprionodon sp. & 4 & $5,3 \%$ & 1 & 3 & 3 & 1 \\
\hline Sphyrna lewini & 19 & $25,0 \%$ & 15 & 4 & 12 & 7 \\
\hline Sphyrna mokarran & 10 & $13,2 \%$ & 10 & $\mathbf{0}$ & 5 & 5 \\
\hline Total & 76 & $100,0 \%$ & 47 & 29 & 43 & 33 \\
\hline
\end{tabular}

TABELA 5: Espécie, número de indivíduos e frequência de tubarões do gênero Sphyrna adquiridos no litoral de Sergipe.

\begin{tabular}{lcccc}
\hline \multicolumn{1}{c}{ Espécie } & № de Indivíduos & $\begin{array}{c}\text { Frequência } \\
\text { \% }\end{array}$ & $\begin{array}{c}\text { № de Indivíduos } \\
\text { Frescos }\end{array}$ & $\begin{array}{c}\text { № de Indivíduos da } \\
\text { Coleção }\end{array}$ \\
\hline Sphyrna tiburo & 5 & 12,8 & 0 & 5 \\
Sphyrna mokarran & 11 & 28,2 & 7 & 4 \\
Sphyrna lewini & 23 & 59,0 & 18 & 5 \\
\hline Total & $\mathbf{3 9}$ & $\mathbf{1 0 0 , 0}$ & $\mathbf{2 5}$ & $\mathbf{1 4}$ \\
\hline
\end{tabular}


representando $59 \%$ da amostra. Os animais frescos somaram 25 exemplares distribuídos em duas espécies: $S$. lewini e $S$. mokarran, que representaram $64,1 \%$ do total de animais analisados.

A frequência de ocorrência por espécie em relação ao sexo mostra que para as espécies $S$. mokarran e $S$. lewini a maior ocorrência foi de machos, e para S. tiburo, representado somente por animais fixados, foi de fềmeas (Figura 6).

Na Figura 7, é possível observar a distribuição dos exemplares quanto ao estágio de desenvolvimento sexual, e, para as três espécies estudadas, nenhum adulto foi capturado. S. mokarran e $S$. lewini apresentaram maior ocorrência de neonatos, e $S$. tiburo, de juvenis com $60 \%$.

Quando se compara o sexo e estágio de desenvolvimento sexual entre espécies, observa-se que $S$. tiburo não obteve machos juvenis, mas obteve uma alta frequência para fềmea juvenil. Já $S$. mokarran e $S$. lewini apresentaram maiores frequências para machos neonatos e menor para fềmeas juvenis (Figura 8).

Os comprimentos peitoral-pélvico (Figura 9) e pélvico-anal (Figura 10) têm bastante relação com o sexo dos animais. Normalmente, as fêmeas apresentam um maior comprimento peitoral-pélvico, uma vez que essa região tem que ser maior para suportar a gestação,

FIGURA 6: Frequência de espécies do gênero Sphyrna por sexo.

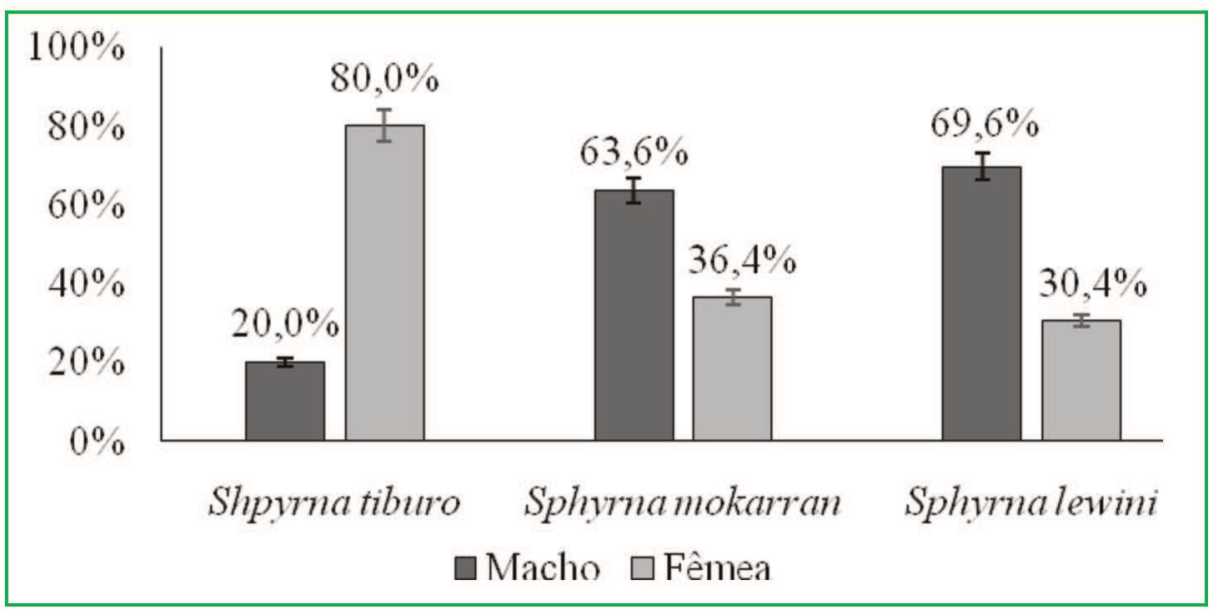

FIGURA 7: Frequência de espécies do gênero Sphyrna por estágio de desenvolvimento sexual.

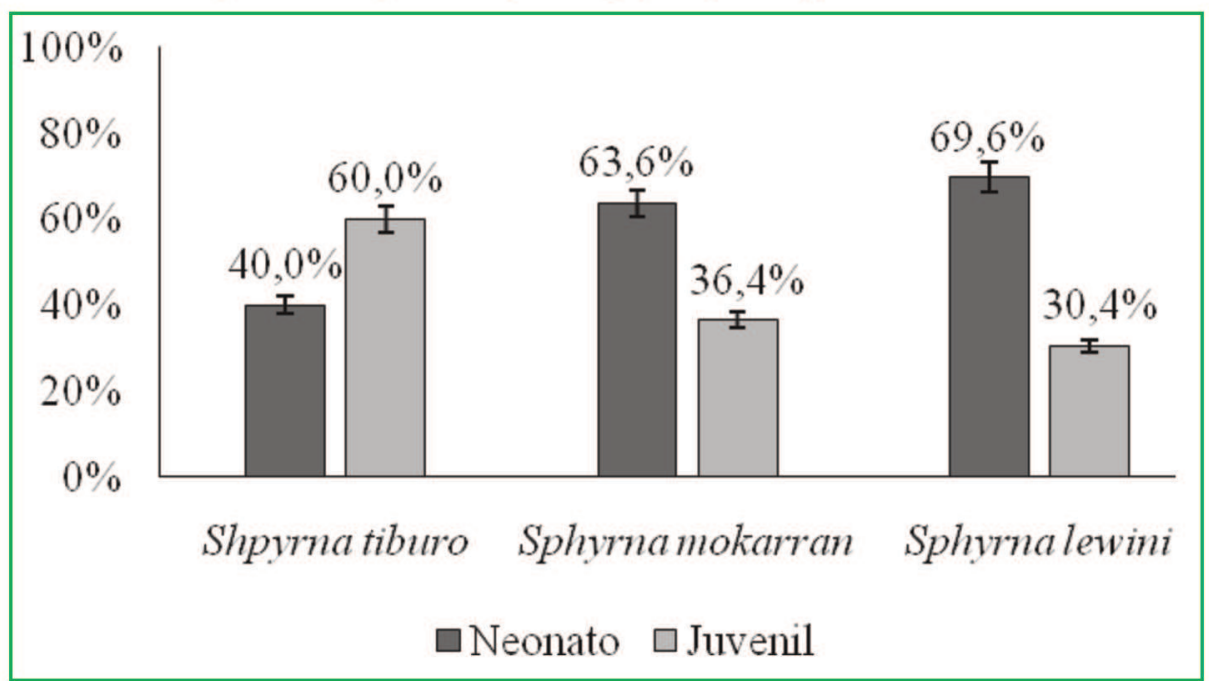


FIGURA 8: Frequência de espécies do gênero Sphyrna por sexo e estágio de desenvolvimento sexual.

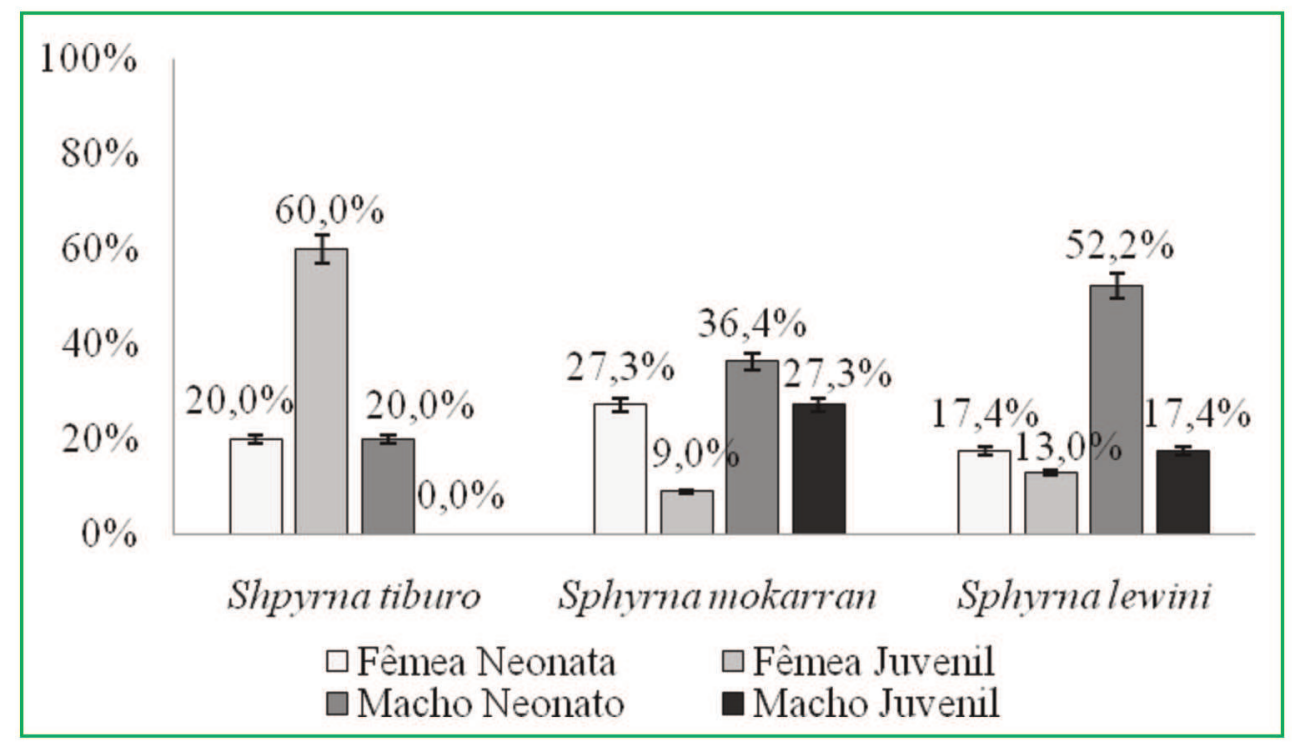

FIGURA 9: Comprimento peitoral-pélvico médio (cm) para ambos os sexos dos espécimes frescos.

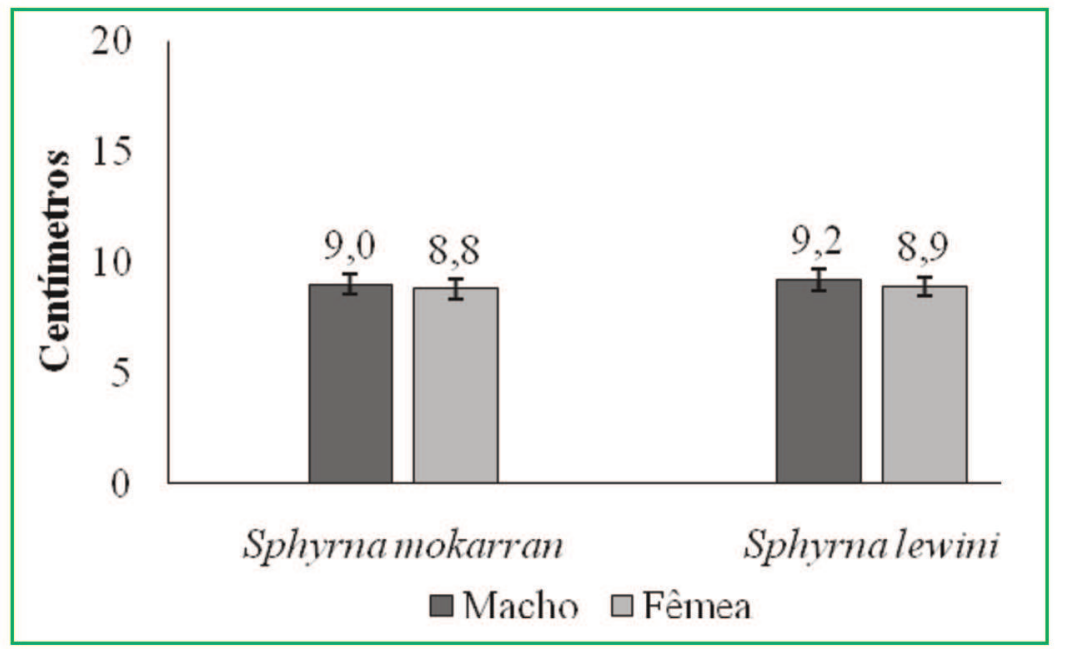

FIGURA 10: Média do comprimento pélvico-anal em relação ao sexo dos animais frescos.

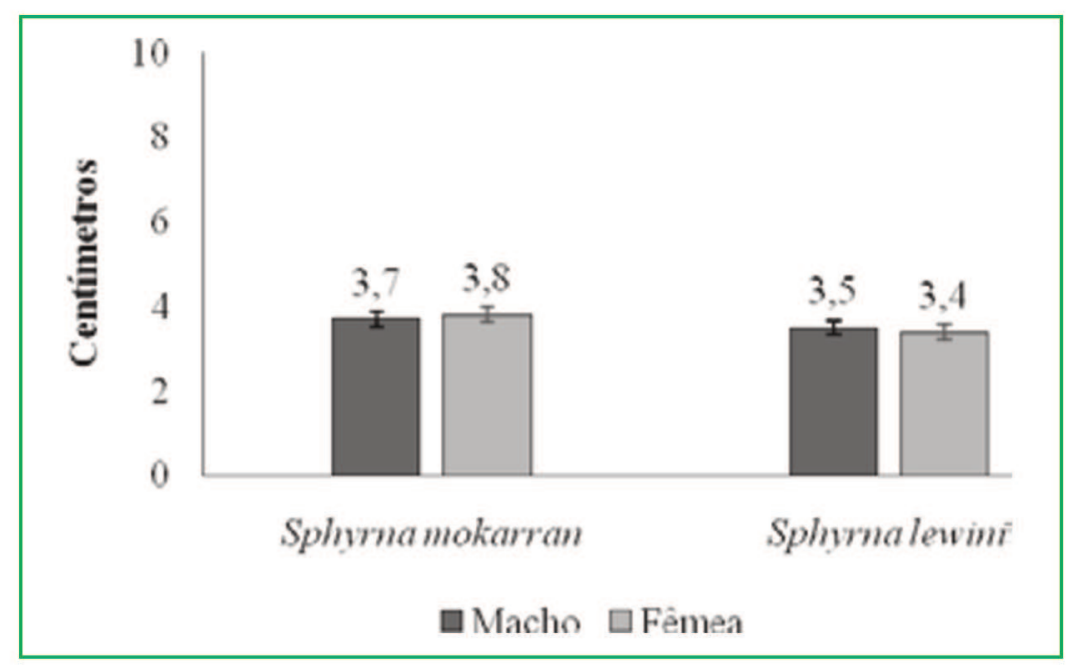


enquanto que para os machos a região pélvico-anal é normalmente maior devido ao desenvolvimento do clásper, estrutura reprodutiva (BASS, 1973).

Em relação ao comprimento pélvico-anal, que normalmente é maior em machos, também não houve diferença significativa entre sexos (Tabela 6).

TABELA 6: Valor de $\mathrm{p}<0,05$ encontrado pelo Teste-T de Student para medidas de comprimento peitoral-pélvico e pélvico-anal.

\begin{tabular}{lcc}
\hline \multicolumn{1}{c}{ Dados Biométricos } & $\boldsymbol{t}$ & $\boldsymbol{P}$ \\
\hline Comprimento peitoral-pélvico & 0,9426 & 0,3556 \\
Comprimento pélvico-anal & 0,3761 & 0.7103 \\
\hline
\end{tabular}

Na Figura 11, pode-se observar a variação do peso de ambas as espécies e a variação do comprimento total.
Pode-se observar que para S. mokarran o maior peso coincide com o maior comprimento total, representado como SM11. Enquanto que para S. lewini, o mesmo não ocorreu, sendo o maior peso para o exemplar SL19 e o maior comprimento total para o SL20.

$\mathrm{Na}$ Tabela 7, encontra-se a variação para o comprimento total das espécies analisadas. A média geral foi semelhante para ambas as espécies.

A média da largura da cabeça (Tabela 8) foi maior para o S. lewini, bem como sua relação com o comprimento total, apesar de essa espécie apresentar um menor comprimento total.

Para o comprimento interdorsal, as espécies estudadas apresentaram valores muito próximos (Figura 12).

FIGURA 11: Valores encontrados para peso e comprimento total de todos os espécimes frescos analisados.

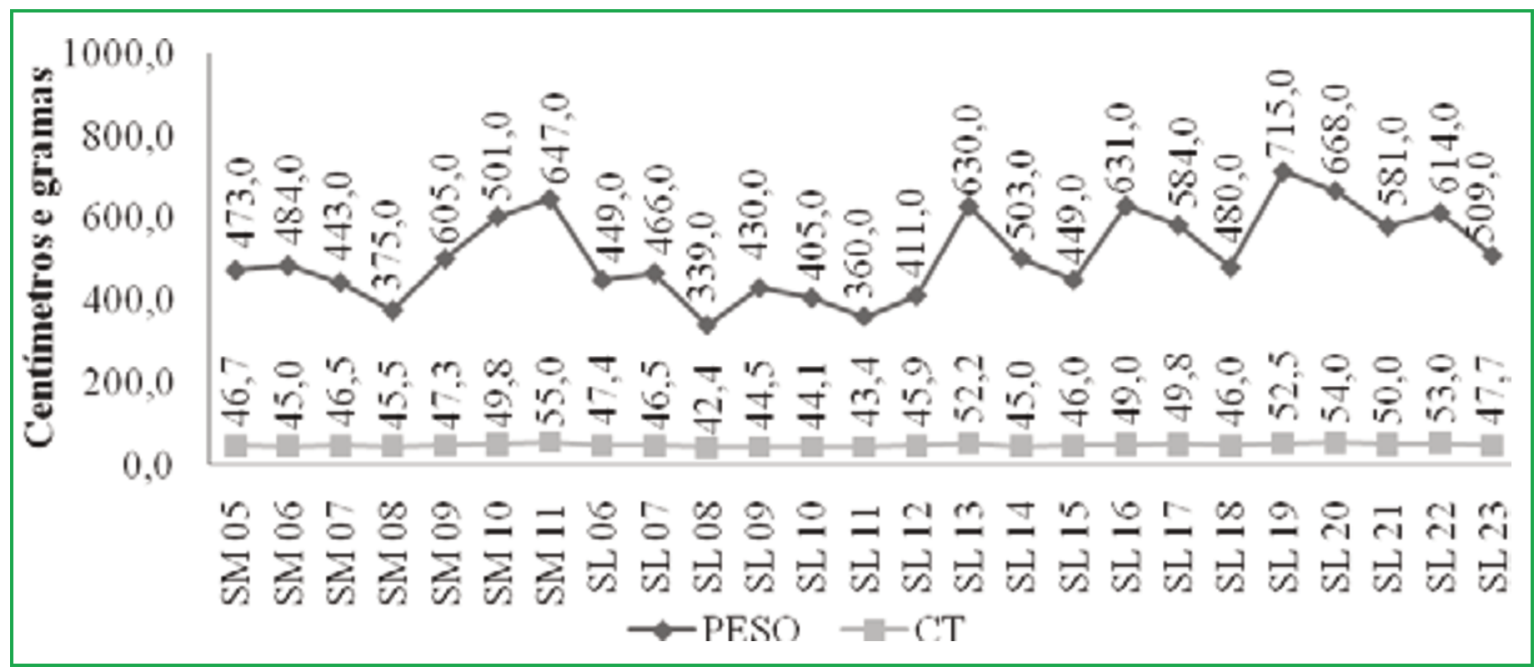

TABELA 7: Média geral por espécie, por sexo e os respectivos valores de mínimo e máximo do Comprimento total para as espécies frescas analisadas.

\begin{tabular}{lccccccc}
\hline \multicolumn{1}{c}{ Espécie } & $\begin{array}{c}\text { Média } \\
\text { Geral }\end{array}$ & $\begin{array}{c}\text { Média } \\
\text { Macho }\end{array}$ & $\begin{array}{c}\text { Média } \\
\text { Fêmea }\end{array}$ & $\begin{array}{c}\text { Mínimo } \\
\text { Macho }\end{array}$ & $\begin{array}{c}\text { Máximo } \\
\text { Macho }\end{array}$ & $\begin{array}{c}\text { Mínimo } \\
\text { Fêmea }\end{array}$ & $\begin{array}{c}\text { Máximo } \\
\text { Fêmea }\end{array}$ \\
\hline Sphyrna mokarran & 48,0 & 47,3 & 48,9 & 45,5 & 49,8 & 45,0 & 55,0 \\
Sphyrna lewini & 47,7 & 48,1 & 47,0 & 42,4 & 53,0 & 43,4 & 54,0 \\
\hline
\end{tabular}

TABELA 8: Média da largura da cabeça e sua relação com o comprimento total de cada espécie analisada.

\begin{tabular}{lccc}
\hline \multicolumn{1}{c}{ Espécie } & Largura da cabeça & Comprimento total & $\begin{array}{c}\text { Relação } \\
\text { largura da cabeça } \times \text { comprimento total }\end{array}$ \\
\hline Sphyrna mokarran & 12,8 & 48,0 & $26,67 \%$ \\
Sphyrna lewini & 13,2 & 47,7 & $27,67 \%$ \\
\hline
\end{tabular}


FIGURA 12: Comprimento interdorsal dos animais frescos analisados.

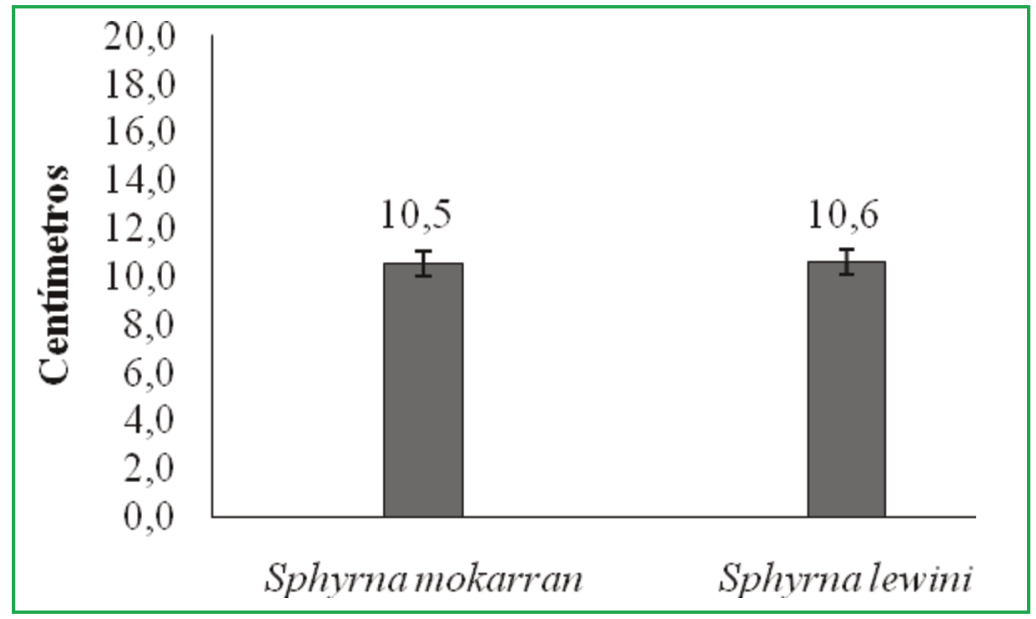

TABELA 9: Valor de $p<0,05$ encontrado pelo Teste-t de Student para a medida de comprimento total e pelo Teste U de Wilcoxon para as medidas da largura da cabeça e comprimento interdorsal.

\begin{tabular}{lccc}
\hline Dados Biométricos & $\boldsymbol{t}$ & $\boldsymbol{u}$ & $\boldsymbol{P}$ \\
\hline Comprimento total & 0,1455 & - & 0,8856 \\
Largura da cabeça & - & 48,50 & 0,3802 \\
Comprimento interdorsal & - & 61,50 & 0,9277 \\
\hline
\end{tabular}

\section{Discussão}

$\mathrm{O}$ gênero estudado obteve uma representatividade de $38,2 \%$ na pesca, sendo a maior frequência para a espécie Sphyrna lewini, com mais indivíduos machos e neonatos. Esta pesca característica de mais indivíduos de S. lewini é comentada por Lessa e Nóbrega (2000) em estudo feito na região Nordeste.

$\mathrm{O}$ maior número de indivíduos para neonatos e machos se assemelha a estudo recente feito por Dolphine (2014), na região de São Paulo, para a espécie S. lewini, em que usou-se o mesmo tipo de embarcação de pequeno porte, mas não o mesmo artefato pesqueiro. Motta (2006) relata a ausência de exemplares adultos na pesca em que se utilizam redes de menores tamanhos entre nós, ou seja, com malha mais estreita, e com isso mais frágeis para comportar animais de maior porte. Além disso, é importante considerar a duração do tempo $(4 \mathrm{~h})$ que a rede fica exposta e sua profundidade, que, no presente estudo, se coloca a poucos metros da superfície (4-8 m) e em um período em que a presença de adultos perto da costa é nula.

Meneses (2008) utilizou em sua pesquisa, no litoral de Sergipe, três artefatos de pesca diferentes, que apresentam um maior alcance de profundidade. Durante maio de 2002 e dezembro de 2007 coletou ao todo 1.514 tubarões, no entanto os tubarões do gênero Sphyrna representaram apenas $3,43 \%$, sendo também $S$. lewini a espécie mais abundante.

Segundo esses estudos de Meneses (2008), em relação ao sexo, a captura das espécies $S$. lewini e S. mokarran obteve mais indivíduos machos, enquanto que a de $S$. tiburo obteve mais fêmeas. Esta última, segundo Lessa et al. (1999), é a espécie de menor porte que ocorre na costa de Sergipe, ao passo que as outras duas, de maior porte, são encontradas mais em regiões oceânicas, adentrando a costa principalmente no litoral das regiões Sul, Sudeste e Nordeste para completar seu ciclo reprodutivo.

A presença de indivíduos neonatos (maioria) e juvenis pode estar relacionada ao padrão de distribuição das espécies, em que fêmeas invadem a costa no período gestacional para parir seus filhotes nessas áreas mais rasas, entre 2 a $10 \mathrm{~m}$, até que atinjam a maturidade sexual e possam então sair das regiões costeiras e partir para maiores profundidades na plataforma continental e oceânica (LESSA; MENNI, 1998; VOOREN et al., 2005). 
Estudos de Meneses (2008) e Dolphine (2014) com pesca artesanal de barcos de pequeno porte e redes diferentes mostraram uma semelhança na captura de neonatos e juvenis. Meneses (2008) apresentou em sua pesquisa dados de três diferentes tipos de redes (emalhar de fundo, emalhar de superfície e espinhel de fundo) que apresentam diferentes alcances de profundidade, e o número maior de exemplares juvenis pode ser devido a esse alcance do petrecho de captura.

Uma relação feita por Kotas (1996) é entre o sexo e o estágio de desenvolvimento sexual, que mostra um crescimento mais rápido das fêmeas, já que apresentam uma dieta bastante generalista, provocando assim sua saída mais rápida da zona costeira. Baseando-se nessa explicação, pode-se notar que os dados obtidos no presente estudo não foram muito diferentes, sendo a captura de fêmeas inferior à de machos juvenis. Contudo, $S$. tiburo foi a única espécie que apresentou o contrário.

De acordo com o comprimento total das espécies, Horn (2014) apresentou dados de comprimento total para neonatos e juvenis da espécie $S$. lewini, com uma média de 55,7 cm e mínimo e máximo de 32,6 e 95,0 cm. Comparando com o presente trabalho, o valor referente à média foi próximo, enquanto as variações mínimas e máximas mais distantes. Ainda segundo Horn (2014) essas medidas se encontram dentro dos parâmetros de comprimento para neonatos.

Segundo Clarke (1971), Castro (1993) e Horn (2014), no comprimento total de neonatos não há muita variedade por causa do pouco tempo que permanecem nesse estágio, entre quatro a seis semanas, sendo logo então classificados como juvenis e passando a crescer uma média de cinco $\mathrm{cm}$ por mês.

A ausência de $S$. tiburo nas capturas torna-se um fato preocupante, tendo em vista que em estudos anteriores no litoral sergipano, realizados por Araújo et al. (1995), a espécie foi uma das mais capturadas.

De acordo com Gadig (2001), a largura da cabeça para a espécie $S$. lewini é em torno de 24 a $30 \%$ do comprimento total, e para $S$. mokarran, de 23 a $27 \%$. No presente trabalho foi observada uma média de 27,6\% e $26,6 \%$ para $S$. lewini e $S$. mokarran, respectivamente.
A falta de mais estudos e pesquisas comparativas sobre as características analisadas associadas à ausência de indivíduos adultos na amostra não possibilitou uma discussão muito ampla desses resultados. Contudo, os dados apresentados no presente trabalho foram semelhantes aos estudos de Gadig (2001), Motta (2006), Meneses (2008), Dolphine (2014) e Horn (2014), com uma maior frequência de indivíduos da espécie Sphyrna lewini, neonatos e machos.

Com relação às características analisadas para as espécies capturadas frescas, o comprimento pélvicoanal foi maior para os machos, conforme encontrado na literatura. Para as análises por espécie, S. lewini apresentou os maiores valores para comprimento total e peso, e maior média para a largura da cabeça e espaço interdorsal. A ausência de significância entre as espécies provavelmente foi influenciada pelo pequeno tamanho da amostra. Deste modo, fica evidente a importância de novas pesquisas e divulgação dos resultados para complementar o banco de informações sobre esse gênero, tendo em vista que a discriminação de espécies de formas semelhantes, mas que constituem entidades biológicas diferentes, é crucial para sua_biodiversidade, exploração e manejo pesqueiro (SILVA, 2014).

\section{Agradecimentos}

Agradecemos a todos que contribuíram com a pesquisa, à Prof ${ }^{\mathrm{a}}$ Andressa Coelho, nossa orientadora, ao Thiago Meneses, por nos proporcionar conhecimento a respeito da área e auxílio no desenvolvimento do trabalho, ao Laboratório do Grupo de Estudo de Elasmobrânquios de Sergipe e seus estagiários, ao curso de Ciências Biológicas da Universidade Tiradentes e ao Laboratório de Biologia Tropical do Instituto de Tecnologia e Pesquisa, pela infraestrutura e apoio logístico. E, por fim, aos pescadores, que forneceram os tubarões ao longo do estudo, além de importantes informações.

\section{Referências}

ARAUJO, M. L. G.; SILVA, V. C.; SILVA, A. C. C. Resultados preliminares do estudo sobre elasmobrânquios capturados ao longo 
da Reserva Biológica de Santa Isabel, Pirambu - SE. In: REUNIÃO DO GRUPO DE TRABALHO SOBRE PESCA E PESQUISA DE TUBARÕES E RAIAS NO BRASIL, VII, 1995, Rio Grande. Anais... Rio Grande: FURG, 1995. p. 22.

BASS, A. J. Analysis and description of variation in the proportional dimensions of Scyliorhinid, Carcharhinid and Sphyrnid sharks. Investigational Report no 32. Durban: Oceanographic Research Institute, 1973. 28 p.

BEZERRA, N. P. A. Deslocamentos verticais e horizontais do tubarão-martelo Sphyrna lewini (Griffith \& Hamilton Smith, 1834) monitorados a partir do arquipélago de São Pedro e São Paulo, Brasil. 2013. 68 f. Dissertação (Mestrado em Recursos Pesqueiros e Aquicultura) - Universidade Federal Rural de Pernambuco, Recife. 2013.

BONFIL, R. Overview of world elasmobranch fisheries. Technical paper, nํ3 341. Rome: FAO Fisheries, 1994.

BRASIL. Portaria no 445, de 17 de dezembro de 2014. Lista nacional oficial de espécies da fauna ameaçadas de extinção Peixes e invertebrados aquáticos. Brasília: Diário Oficial da União, no 245, 18 dez. 2014. Seção 1.

CASTRO, J. I. The shark nursery of Bulls Bay, South Carolina, with a review of the shark nurseries of the southeastern coast of the United States. Environmental Biology of Fishes, New York, v. 38, p. 37-48, 1993.

CLARKE, T. A. The ecology of the scalloped hammerhead shark, Sphyrna lewini, in Hawaii. Pacific Science, Honolulu, v. 25, p. 133144, 1971.

COMPAGNO, L. J. V. An annotated and illustrated catalogue os shark species known to date. Carcharhiniformes. Vol. 4, Part. 2. In: FAO (Ed.). FAO Fisheries Catalogue. Rome: FAO Fish Synop (125), 1984. p. 251-655.

COMPAGNO, L. J. V. An annotated and illustrated catalogue os shark species known to date. Vol. 2. In: FAO (Ed.). Sharks of the world. FAO, Rome, 2001. 269 p.

DOLPHINE, P. M. Hábitos alimentares de jovens de tubarãomartelo Sphyrna lewini (Griffith \& Smith, 1834) (Condrichthyes, Sphyrnidae) no litoral do estado de São Paulo. 2014. $138 \mathrm{f}$. Dissertação (Mestrado em Zoologia) - Universidade Estadual Paulista "Júlio de Mesquita Filho, Rio Claro, 2014.

FIGUEIREDO, J. L. Manual de peixes marinhos do sudeste do Brasil. São Paulo: Museu de Zoologia, Universidade de São Paulo, 1977. 108 p.

GADIG, O. B. F. Fauna de tubarões da costa Norte/Nordeste do Brasil (Chondrichthyes, Elasmobranchii). 1994. $279 \mathrm{f}$. Dissertação (Mestrado em Zoologia) - Universidade Federal da Paraíba, João Pessoa. 1994.

GADIG, O. B. F. Tubarões da costa brasileira. 2001. 360 f. Tese (Doutorado em Zoologia) Universidade Estadual Paulista Júlio de Mesquita Filho, Rio Claro. 2001.

GEES. Grupo de Elasmobrânquios de Sergipe. 2013. Disponível em: <htpp: www.elasmosergipe.blogspot.com.br/>. Acesso em: 12 abril 2015.

GILBERT, C. R. A revision of the hammerhead sharks (family Sphyrnidae). Smithsonian Institution, Washington, v. 119, n. 3539, p. 1-88, 1967.
HORN, T. S. Aspectos biológicos de Sphyrna lewini (Griffith \& Smith, 1834) no litoral norte do Rio Grande do Sul: subsídios para a identificação dos seus berçarios. 2014. 53 f. Monografia (Bacharelado em Ciências Biológicas) - Universidade Federal do Rio Grande do Sul, Imbé/Osório. 2014.

IUCN. União Internacional para a Conservação da Natureza. 2015. Disponível em: <http://www.iucnredlist.org/> Acesso em: 14 abril 2015.

KOTAS, J. E. Relatório preliminar sobre a biologia e pesca do tubarão-martelo Sphyrna lewini (Griffith \& Smith) no mar territorial e Zona Econômica exclusiva do sul do Brasil durante os anos 1995-1996. Itajai: CEPSUL/IBAMA, 1996. 22 p.

KOTAS, J. E. Dinâmica de populações e pesca do tubarãomartelo Sphyrna lewini (Griffith \& Hamilton Smith, 1834), capturado no mar territorial e zona econômica exclusiva do sudeste-sul do Brasil. 2004. 419 f. Tese (Doutorado em Ciências da Engenharia Ambiental) - Escola de Engenharia de São Carlos, Universidade de São Paulo, São Carlos. 2004.

LESSA, R. P.; MENNI, R. C. The chondrichthyan community off Maranhão (northeastern Brazil). Acta Zoologica Lilloana, Tucumán, v. 44, n. 1, p. 69-89, 1998.

LESSA, R.; NÓBREGA, M. F. de. Guia de identificação de peixes marinhos da Região Nordeste. Recife: Programa REVIZEE, 2000. $138 \mathrm{p}$.

LESSA, R.; SANTANA, F. M.; RINCÓN, G.; GADIG, O. B. F.; EL-DEIR, A. C. N. Biodiversidade de Elasmobrânquio no Brasil. Recife: Ministério do Meio Ambiente - Projeto de Conservação e utilização Sustentável da Diversidade Biológica Brasileira (PROBIO), 1999. 154 p.

MENESES, T. S. Fauna, pesca e contaminação por metais pesados em pescado de tubarões no litoral de Sergipe. 2008. 118 f. Dissertação (Mestrado em Saúde e Ambiente) - Universidade Tiradentes, Aracaju. 2008.

MENESES, T. S.; PEREIRA, C. W.; SANTOS, F. N. Pequenos tubarões costeiros capturados por espinhel de fundo operado por embarcação artesanal no litoral de Sergipe. Arquivos Ciências do Mar, Fortaleza, v. 44, n. 1, p. 47-52, 2011.

MOTTA, F. S. Ecologia e pesca artesanal de tubarões costeiros no litoral centro-sul de São Paulo. 2006. 172 f. Tese (Doutorado em Zoologia) - Universidade Estadual Paulista, Rio Claro. 2006.

QUATTRO, J. M; DRIGGERS III, W. B; GRADY, J. M; ULLRICH, G. F; ROBERTS, M. A. Sphyrna gilberti sp. nov., a new hammerhead shark (Carcharhiniformes, Sphyrnidae) from the western Atlantic Ocean. Zootaxa, Auckland, v. 3702, n. 2, p. 159178, 2013.

SANTOS. P. P.; VILAR. J. W. C. Planejamento territorial turístico do litoral sergipano. Revista Geonorte, Manaus, v. 3, n. 4, p. 1194 1206, 2012.

SADOWSKY, V. The hammerhead sharks of the littoral zone of São Paulo, Brazil, with the description of a new species. Bulletin of Marine Sciences, Miami, v. 15, n. 1, p. 1-12, 1965.

SBEEL - SOCIEDADE BRASILEIRA PARA O ESTUDO DE ELASMOBRÂNQUIOS. Plano Nacional de ação para a conservação e o manejo dos estoques de peixes elasmobrânquios no Brasil. Recife: SBEEL, 2005. 99 p. 
SILVA, R. M. de S. Diferenças morfométricas de duas espécies do gênero Rhizoprionodon (Chondrichthyes, Carcharhinidae). 2014. 48 f. Dissertação (Mestrado em Biometria e Estatística Aplicada) - Universidade Federal Rural de Pernambuco, Recife. 2014.

SIMPFENDORFER, C. A.; MILWARD, N. E. Utilization of a tropical bay as a nursery area by sharks of the families Carcharhinidae and Sphyrnidae. Environmental Biology of Fishes, New York, v. 37, p. 337-345, 1993.
SOTO, J. M. R. Annotated systematic checklist and bibliography of the coastal and oceanic fauna of Brazil. I. Sharks. Mare Magnum, Itajaí, v. 1, n. 1, p. 51-120, 2001

VOOREN, C. M.; KLIPPEL, S.; GALINA, A. B. Biologia e status da conservação dos tubarões-martelo Sphyrna lewini e $S$. zygaena. In: VOOREN, C. M.; KLIPPEL, S. (Ed.). Ações para a conservação de tubarões e raias no sul do Brasil. Porto Alegre: Igarapé, 2005. p. 97-112. 